\title{
The Algorithms of Preliminary Processing of the Inclinometric Control Data during the Monitoring of the Building
}

\author{
PhD student, A.V. Grecheneva ${ }^{1,3}$ D.Sc. in Engineering, Professor, O.R. Kuzichkin ${ }^{1}$ Ph.D., associate Professor N.V. \\ Dorofeev $^{2}$ Ph.D., associate Professor D.I. Surzhik ${ }^{2}$
}

${ }^{1}$ Belgorod State University, 85 Pobedy St, Belgorod, 308015, Russia

${ }^{2}$ Vladimir State University, 87 Gor'kogo St, Vladimir, 600000, Russia

Corresponding author; E-mail: 1155464@bsu.edu.ru

\begin{abstract}
The article is devoted to the development of algorithmic support of the inclinometric control system built on the basis of accelerometers using the phase-metric method. This system is used to automated control the deviation of the angular parameters of the building axis from the vertical. An algorithm for data collection and transmission has been developed that allows automatic data collection from the accelerometer network via the $802.11 \mathrm{~b} / \mathrm{h} / \mathrm{g}$ interface. An algorithm for calculating the angle of deviation of the building axes from the vertical is developed on the basis of the phase-metric method. According to this algorithm for determining the angle, it is necessary to register the moments of crossing the reference signal zero and the signal of the accelerometers are fixed along the construction axes. The time difference of the zero values of the signals in each of the periods will be equal to the deviation angle of the building axis from the vertical. To implement the phase-metric method of angle measurement, an algorithm is developed for the sub-program of the zerocrossing detector. This algorithm describes the sequence of operations of comparing signals for the transition of the value of the signal parameters from positive to negative and vice versa. In the final section, the error of the developed software implemented in the experimental inclinometer installation was estimated.
\end{abstract}

\section{Introduction}

Inclinometric control is an integral part of the systems of geotechnical monitoring of buildings and structures [1]. Monitoring the tilt of buildings and structures involves recording the angle of deviation of the building axes from the vertical along with the monitoring of foundations (their sediment) [2]. Objects are built on problem soils (such as bulk soil, permafrost soil, swelling soil, subject to karst phenomena) must in any case be listed as objects to be monitored for the tilt of buildings and structures. If during the construction (or subsequent operation) the defects were detected on any objects (are not included in the mandatory list), then those objects are also monitored. A separate class are life support facilities and increased danger (such as nuclear reactors), which should be monitored continuously. Sanitary norms and rules 3.03.01-87 "Bearing and enclosing structures" (RU) establish requirements for the accuracy of the measurements.

For conducting measurements, autonomous inclinometers are used. The existing monitoring tools allow tracking changes in the spatial state and geometric parameters of the structure (tiltmeters) equipped with various types of sensors: a solid-state accelerometer, a compensated servo accelerometer, an electric converter DTE, etc. [3].

The requirements for the accuracy and reliability of the measurement information obtained with modern measuring inclinometers are very stringent, which determines the increased conditions for their metrological characteristics and reliability characteristics. The solution of this problem is the system of inclinometric control developed by the authors of this article on the basis of accelerometers [4-6].
The aim of this work is the development of algorithmic support of the inclinometric control system using the phase-metric method at the stage of primary data processing, which will allow increasing the accuracy and noise immunity of the measuring system.

\section{The Algorithm for Calculating the Tilt Angle on the Basis of the Phase-Metric Method}

The application of phase-metric method is based on recording the phase difference of harmonic oscillations [7]. In this case, the functional dependencies are described in the form of a vector model of oscillatory motion (Fig. 1).

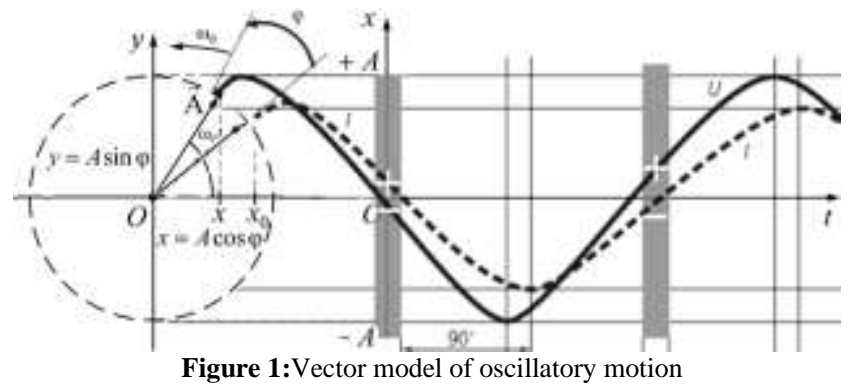

Since the phase is a quantity describing the oscillatory process and representing the argument of the sinusoidal time function $[8,9]$, then in the presence of two harmonic signals $U_{1}=U \cdot \sin \left(\omega_{1} t+\varphi_{1}\right)$ and $U_{2}=U \cdot \sin \left(\omega_{2} t+\varphi_{2}\right)$ 
with different frequencies, the phase difference is a linear time function and is determined based the equation:

$\Delta \varphi=\left(\omega_{1}-\omega_{2}\right) t+\left(\varphi_{1}-\varphi_{2}\right)$.

In the physical sense, the phase difference is the difference in the times at which the phases of each of the oscillations are equal [10]. For example, the formula for the phase difference of two voltage oscillations passing through zero can be obtained on the basis of the difference of the instants of time $t_{i}, t_{i+1}$ and taking into account the equality of the instantaneous phases:

$\Delta \varphi=\varphi_{1}-\varphi_{2}=\omega\left(t_{i+1}-t_{i}\right)=\frac{2 \pi}{T}\left(t_{i+1}-t_{i}\right)$.

In this case, the key characteristic is the delay time:
$\tau=\frac{\Delta \varphi}{2 \pi f}$.

In the developed system of inclinometric control, the processing of accelerometer signals is carried out by use the compensating phase method, the essence of which is the presence of a reference harmonic signal, a comparison with which allows us to determine the phase shift $\varphi_{i}$ [11]. In digital accelerometers, phase measurement is based on the measurement of time intervals (Figure 2).

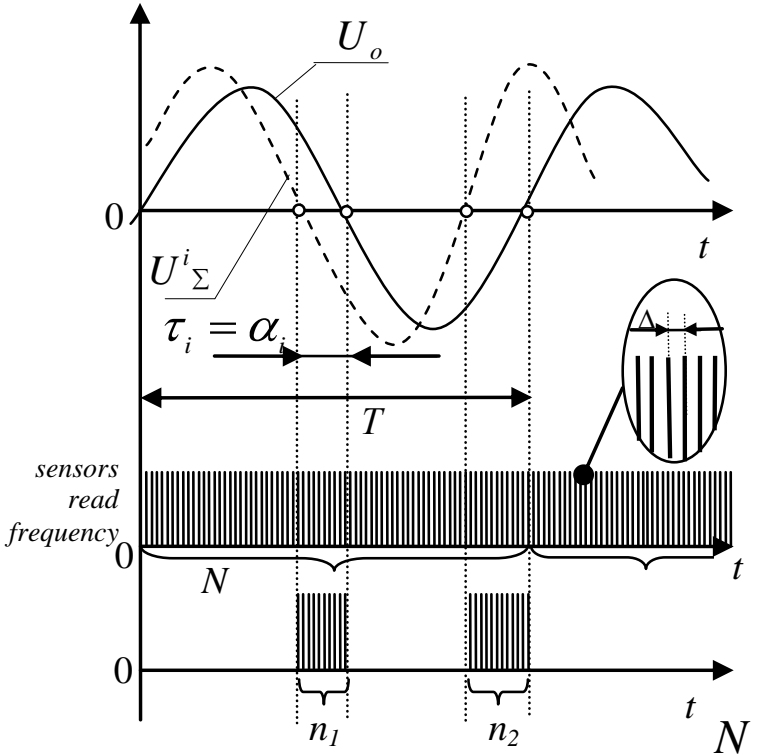

Figure 2: Diagram of digital implementation of the phase-metric method for determining the tilt angle

Generation of the reference sinusoidal signal is based on the frequency of the quartz crystal of the microprocessor that is part of the measuring system. Based on the fact that the accelerometers work with a certain sampling frequency, it is necessary to form counting pulses of sensor interrogation.

To determine the angle, it is necessary to register the moments of zero-crossing the reference signal $U_{o}=\sin \cdot \omega t$ and the accelerometer signal $U^{i}{ }_{\Sigma}$ transformed during the mathematical transformations, the time $\tau_{i}$ difference of the zero values of the signals in each of the periods will be equal to the angle of deviation $\alpha_{i}$ of the axis of the structure from the vertical.

Analytically, the dependence of the number of pulses entering the time interval of the phase shift on the total number of pulses in the period is determined from the expression:

$\frac{n_{i}}{N_{i}}=\frac{\tau_{i}}{T}$.

Taking into account the linear dependence, the angle of rotation is determined by:

$$
\Delta \varphi=360^{\circ} \cdot \frac{\tau_{i}}{T}=360^{\circ} \cdot \frac{n_{i}}{N_{i}} .
$$

Either from the formula:

$$
\Delta \varphi=n_{i} \cdot \Delta \text {, }
$$

where $\Delta$ is the time interval between the samples of the sensors interrogation.

The algorithm for software implementation of the phase-metric principle of measuring the tilt angle between the series-connected accelerometers is presented in Figure 3. This algorithm is a sequential cyclic conversion of signals from accelerometers recorded in real-time measurement arrays.

The total execution time of the main program cycle is determined by the hardware characteristics of the microprocessor included in the measuring system. In this case, the time $\Delta$ determines the time interval between the counts of the sensors interrogation. The condition for the program to close is pressing the reset button, which is polled at the beginning of the main program cycle.

\section{The Algorithm of the Zero Crossing Detector Subroutine}

The execution of the main program algorithm involves the launch of the zero crossing detector subroutine, the block diagram of which is shown in Figure 4 [12-13]. This algorithm describes the sequence of operations of comparing signals for the transition of the value of the signal parameters from positive to negative and vice versa.

The algorithm implies the presence of the intersection flags (Det_U, Det_ $\alpha$, Det_ $\beta$, Det_ $\gamma$ ), which are set to 1 or to 0 , depending on the conditions. This is necessary to synchronize the detection. If there is a zero crossing of the function of the reference signal and one of the functions of the accelerometer sensor (multiplied by the quadrature signal), it is possible to continue checking the execution of the remaining conditions in the given measurement period, relative to the registered reference signal time.

The result of this algorithm is the calculation of the tilt angles $\alpha, \beta, \gamma$ relative to the difference in the zero crossing time of the 
sinusoidal function of the reference oscillator and the harmonic function of the summed signals $S_{\alpha}, S_{\beta}, S_{\gamma}$.

\section{The Algorithm for Data Collection and Transmission}

To implement data acquisition from LIS331dlh accelerometers based on the ATMega328 microprocessor and the ESP8266 Wi-Fi module, an algorithm was developed, shown in Figure 5. This algorithm includes the sequence of the following steps:

Step 1. Connect of the standard C ++ libraries (stdint.h), the libraries for working with the i2c interface (wire.h), and the libraries for working with the accelerometer modules (lis331dlh.h) and the Wi-Fi module (ESP8266.h). This step allows the use of standard methods of data transmission and reception from the modules according to the protocols they support.

Step 2. Determination of constants and global variables. In this step, you specify the network ID and password of the access point to which you want to connect to the Wi-Fi module, and you also specify a port for setting up the TCP connection. Also, at this step, the addresses of the multiplexer and accelerometer modules are announced, which, based on the characteristics of the LIS331dlh chip, is not dynamic.

Step 3. Running of the installation cycle.

Step 4. Configuring the LED indication ports, allow determining the error type of the inclinometric control system.

Step 5. Open a serial port, implemented on the basis of a serial UART interface for transferring data from the microprocessor to the Wi-Fi module.

Step 6. Running the main program cycle.

Step 7. Performing the setup of the accelerometers connected via the multiplexer of $i 2 \mathrm{c}$ interface to the microprocessor. This setting involves selecting the multiplexer channel, starting the accelerometer located on this channel, setting the sensitivity of the accelerometer. When implementing the inclinometric control system, a 4-channel multiplexer was selected. While the number of channels is less than 4, perform step 7, otherwise go to step 8 .

Step 8. Obtaining the version of the Wi-Fi module. This procedure implements the AT command AT + GMR and returns the firmware version. The version is displayed as 8 digits. The first group of 4 digits is the AT version, the second group 4 is the SDK version.

Step 9. Setting the Wi-Fi module mode as a client. The procedure implements the AT command AT + CWMODE_CUR = $1(1=$ Station (WiFi client)). If the answer "Ok" is received, go to step 10 , otherwise set the counter $\mathrm{k}=1$, turn on the LED, turn off the LED k-times. Then go to step 6.

Step 10. Connecting the Wi-Fi module to the access point. This procedure executes the AT command AT + CWJAP_CUR = <network identifier>, <password>. The result of successful connection to the access point is the return of "Ok" or the connection error "ERROR". If the answer is "Ok", go to step 11, otherwise set the counter to $\mathrm{k}=2$, turn on the LED, turn off the LED k-times. Then go to step 6.

Step 11. Establish a TCP connection, or get information about the current connection, the AT + CIPMUX command $=1$, returns "OK", "ERROR" or "ALREADY CONNECT". If the answer "Ok" is received, go to step 12, otherwise set the counter to $\mathrm{k}=3$, turn on the LED, turn off the LED k-times. Then go to step 6 .

Step 13. Establish a TCP connection (AT + CIPSTART $=\langle$ type $\rangle$, <address>, <port>). If the answer is "Ok", go to step 14, otherwise set the counter to $k=4$, turn on the LED, turn off the LED ktimes. Then go to step 6 .

Step 14. Reading the data from the accelerometers in the memory of the microprocessor using the i2c interface. As long as the number of channels is less than 4 , perform step 14, otherwise go to step 15 .
Step 15. Sending data from the microprocessor via the Wi-Fi module to the TCP server. The procedure executes the AT + CIPSEND command. The data length in the packet is up to 2048 bytes. After receiving this command, the module switches to the UART data reception mode, after receiving the necessary length data, it transmits them to the mux channel. If the transmission succeeds, returns "OK". If the answer is "Ok", go to step 16, otherwise set the counter to $\mathrm{k}=5$, turn on the LED, turn off the LED k-times. Then go to step 6.

Step 16. Closing the TCP connection. The procedure executes the $\mathrm{AT}+$ CIPCLOSE command. Returns "LINK IS NOT" or "UNLINK" if the ID connection is already broken, "ERROR" if there is no connection. Go to step 6 . 


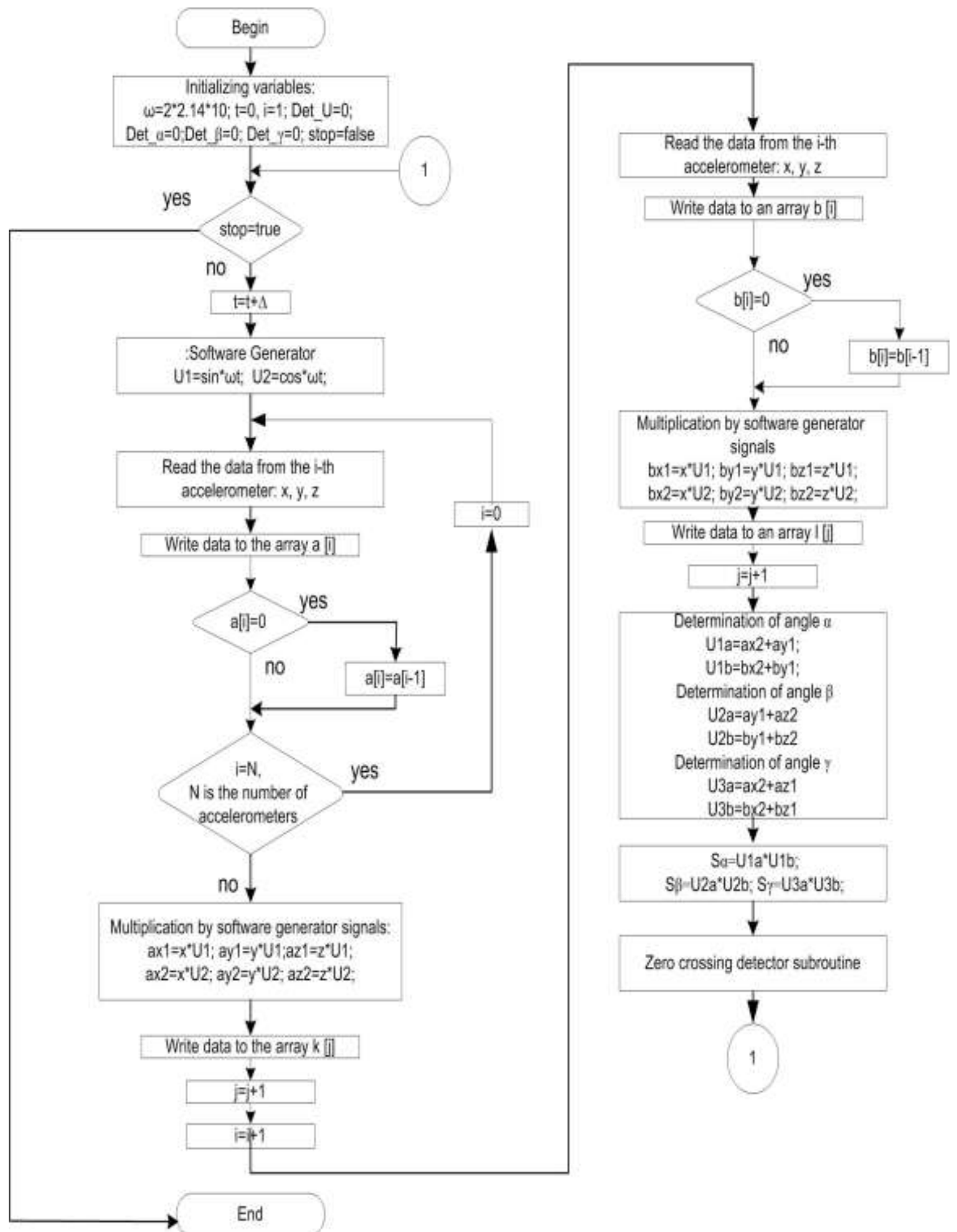

Figure 3::The block diagram of the algorithm for the implementation of inclinometric measurements based on the phase-metric method 


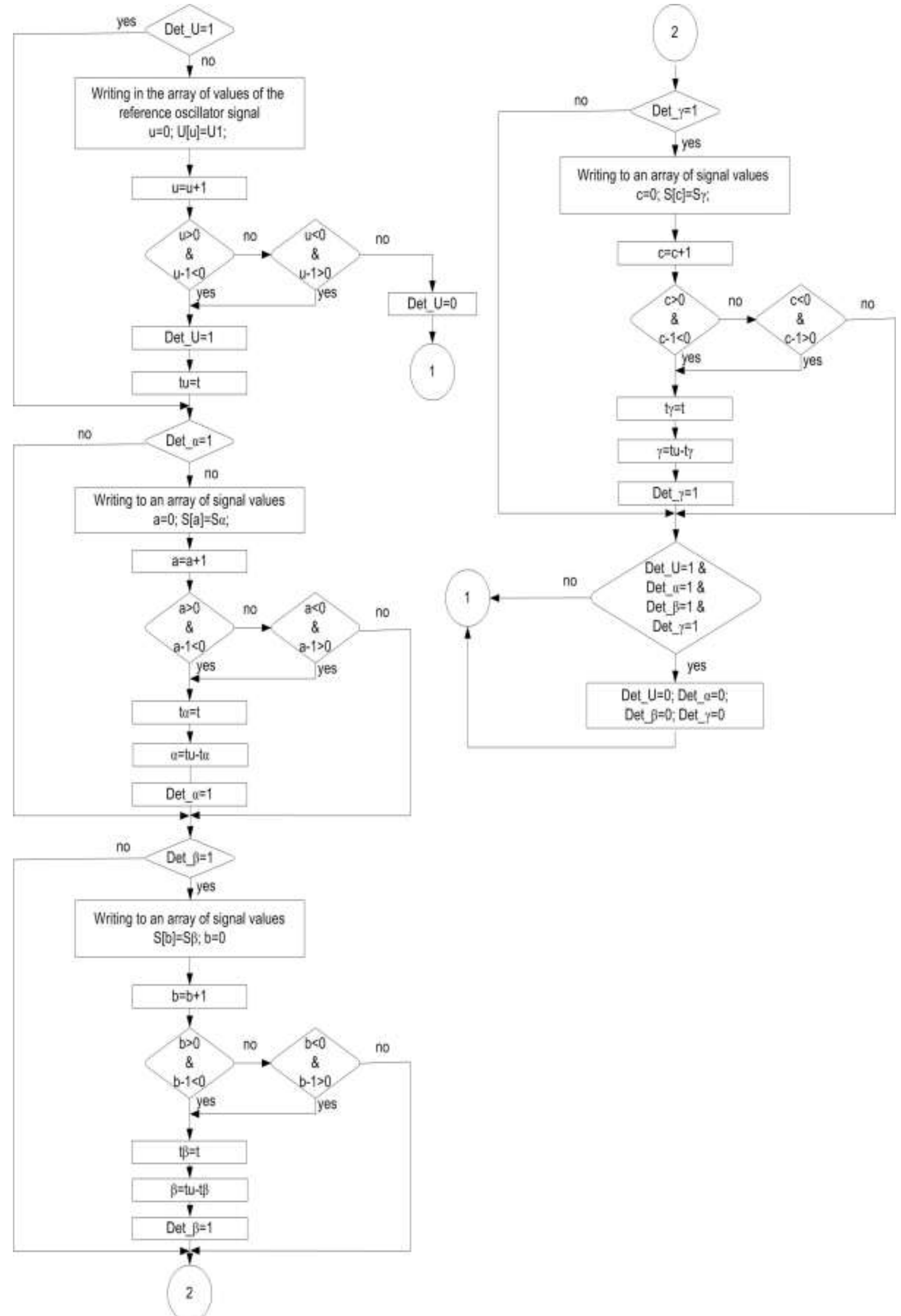

Figure 4: The block diagram of the algorithm of the zero crossing detector subroutine 


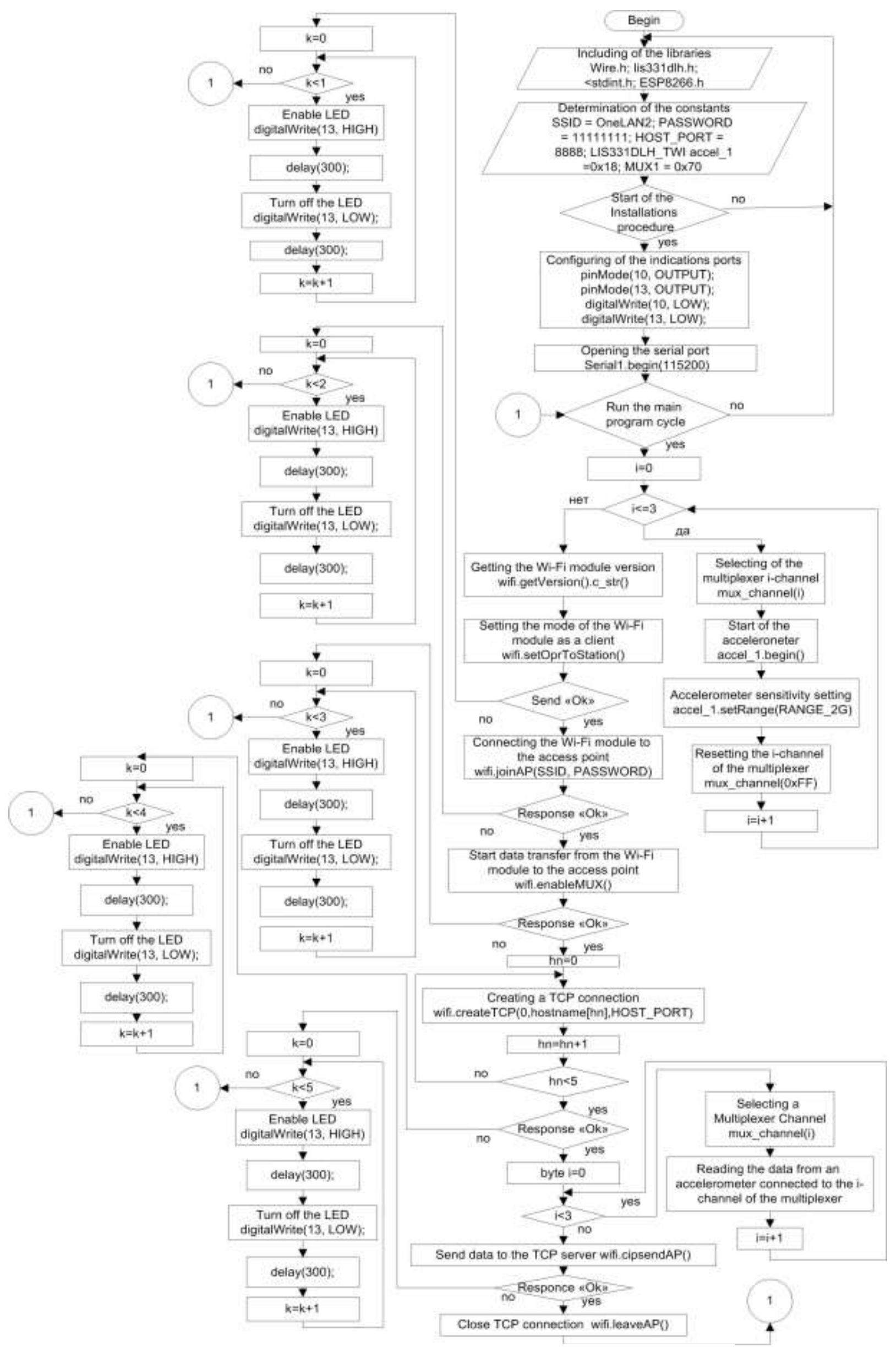

Figure 5: The block diagram of the algorithm for data collection and transmission 


\section{Results and Conclusions}

It is advisable to take into account that the estimation of the software error essentially depends on the specific software and hardware environment in which it is implemented. The error rate is mainly influenced by the clock frequency and processing power of the microprocessor, the complexity (time) of the operations performed, and the transmission rate limitations along the interface lines [14].
As described earlier, the use of the phase-metric method of tilt angle measurement implies the recording of a time interval proportional to the angle of mutual tilt of the accelerometers. While the accelerometer 1 is at position 1 at time $t_{1}$, and the accelerometer 2 at time $t_{1}$ is at position 2 , the angle of mutual deviation is $\varphi$. The error in determining the angle $\varphi$ occurs when the time difference $\tau$ of reading the signal from the accelerometer 1 and reading the signal from the accelerometer 2 increases and the angle between the axis of the accelerometers changes $\varphi=\varphi+\tau$. This is reflected in the software error graph (Figure 6).

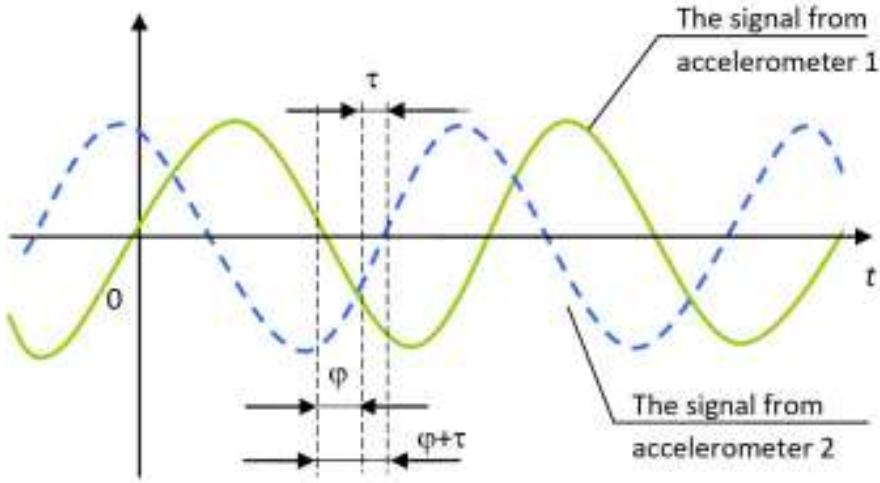

Figure 6: The diagram of software error

In the case of a inclinometric control system based on the phasemetric method, the software error causes temporary delays in the process of reading the data from the accelerometers via the i2c bus to the microcontroller, the procedure for transferring data from the microcontroller to the Wi-Fi module via the UART interface, and the procedure for transferring data to the TCP server with the use of the interface $802.11 \mathrm{~b} / \mathrm{n} / \mathrm{g}$. In this case, each procedure implies sequential execution of functions.

These functions are performed according to the system time of a particular microcontroller. Calculation of the time delay of data transfer within the thesis work is pleasant for a hardware module based on the ATMega328P-PU microcontroller (Table 1).

Table 1: Time for execution of software data transfer functions

\begin{tabular}{|l|l|c|}
\hline Function & Appointment & lead time \\
\hline Wire.read() & Reading the data from the accelerometer & $2 \mu$ s / register \\
\hline Serial.write() & $\begin{array}{l}\text { Data transfer between microprocessor and WiFi module via UART } \\
\text { interface }\end{array}$ & $3.69 \mu \mathrm{s} /$ register \\
\hline Serial.read() & Reading WiFi module signals & $0.65 \mu \mathrm{s} / \mathrm{register}$ \\
\hline
\end{tabular}

When reading the data from the accelerometer, it should be borne in mind that the number of registers is equal to the number of sensitive sensor axes that, in the case of using the accelerometer LIS331dlh, is 3. In order to implement the phase-metric method developed in the thesis, the number of accelerometers in the measuring circuit must be at least 2 . Consequently, the time delay for recording the data of a pair of accelerometers will be $6 \mu \mathrm{s}$, which is not an essential error in the case of monitoring objects whose frequency does not exceed $16 \mathrm{kHz}$ (for high-rise buildings, the fundamental tone of the natural frequency oscillations lies in the range $0.2-0.8 \mathrm{~Hz}$, frequencies above $25-30 \mathrm{~Hz}$ are not advisable because the useful signal is masked by interference). The total time for performing the data reading functions from the sensors is $12 \mu \mathrm{s}$. The data exchange time between the microprocessor and the WiFi module via the UART interface will be $22.14 \mu \mathrm{s}$. The total delay time will be $34.79 \mu \mathrm{s}$.

Thus, the microprocessor realization of the phase-metric method makes it possible to determine the magnitude of the phase shift $\Delta \varphi$ at any chosen reference period. Comparing the phase shifts characteristic for different measurement periods, it becomes possible to estimate the static parameters of the fluctuations $\Delta \varphi$ (mathematical expectation, variance, standard deviation, average value of the phase shift). This feature is most optimal for modeling dynamic processes, as well as for predicting inclinometric changes.

\section{Acknowledgements}

This work was supported in part by the Ministry of Education and Science of the Russian Federation under Grant № 5.3606.2017/ $\mathrm{PCH}$.

\section{Reference}

[1] POSITAL. "MEMS Inclinometer from POSITAL: How do they work?". Posital. Retrieved 2017-01-04.

[2] Rose, Judy (April 21, 2002). "The Hole Story: House construction starts with digging out the basement and pouring its concrete walls". Detroit Free Press. Archived from the original on 2002-0602. Retrieved May 24, 2018.

[3] J. Kuckartza, P. Collier G. Hutchinson a The Design of an Integrated Structural Monitoring System for a High-Rise Building Based on Tiltmeters and GNSS

[4] Kuzichkin O.; Grecheneva A.; Mikhaleva E., Dorofeev N. Carrying out geodeformation monitoring in karst areas based on accelerometric goniometers // Journal of fundamental and applied sciences Vol: 9 Issure: SI pp 1871-1884.

[5] Grecheneva, A.V., Kuzichkin, O.R., Dorofeev, N.V., Eremenko, V.T. Application of a phase-measuring method in the inclinometric systems of geotechnical monitoring // Proceedings of the 2017 IEEE 9th International Conference on Intelligent Data Acquisition and Advanced Computing Systems: Technology and Applications, IDAACS 2017. Volume 1, Pages 168-171.

[6] Kuzichkin, O., Grecheneva, A., Mikhaleva, E., Dorofeev, N., Maxim, B. Application of phase-metric measuring systems for geodynamic control of karst processes // Journal of Engineering and 
Applied Sciences, Volume 12, Issue Specialissue4, 2017, Pages 6858-6863.

[7] Grecheneva, A.V. Application of accelerometers in measuring goniometric systems / A.V. Grecheneva, O.R. Kuzichkin, N.V. Dorofeev, I.S. Konstantinov // Information Systems and Technologies No. 4 (90) 2015 - P. 5-10

[8] Boashash, B., ed. (2003), Time-Frequency Signal Analysis and Processing: A Comprehensive Reference, Oxford: Elsevier Science, ISBN 0-08-044335-4.

[9] Folland, Gerald (1989), Harmonic analysis in phase space, Princeton University Press.

[10] Hewitt, Edwin; Ross, Kenneth A. (1970), Abstract harmonic analysis, Die Grundlehren der mathematischen Wissenschaften, Band 152, Vol. II: Structure and analysis for compact groups. Analysis on locally compact Abelian groups, Springer, MR 0262773.

[11] Kuzichkin O.R., Dorofeev N.V. Elimination of the multiplicative instability of the parameters of differential measuring converters // Methods and devices of information transmission and processing. Вып. 10. - Moscow: Radio Engineering, 2008. - P. 79-82.

[12] Mike Curtin and Paul O'Brien (July-August 1999). "Phase Locked Loops for High-Frequency Receivers and Transmitters-3". Analog Dialogue. Analog Devices. Retrieved 2006-04-25.

[13] Crawford, James A. (1994), Frequency Synthesizer Design Handbook, Artech House, ISBN 0-89006-440-7

[14] MI 2174-91(RU) Certification of algorithms and data processing programs for measurements 